\title{
Arrows as anchors: An analysis of the material features of electric field vector arrows
}

\author{
Elizabeth Gire* \\ Department of Physics, University of Memphis, Memphis, Tennessee 38152, USA
}

Edward Price

Department of Physics, California State University, San Marcos, California 92096, USA

(Received 22 June 2013; revised manuscript received 21 February 2014; published 13 August 2014)

\begin{abstract}
Representations in physics possess both physical and conceptual aspects that are fundamentally intertwined and can interact to support or hinder sense making and computation. We use distributed cognition and the theory of conceptual blending with material anchors to interpret the roles of conceptual and material features of representations in students' use of representations for computation. We focus on the vector-arrows representation of electric fields and describe this representation as a conceptual blend of electric field concepts, physical space, and the material features of the representation (i.e., the physical writing and the surface upon which it is drawn). In this representation, spatial extent (e.g., distance on paper) is used to represent both distances in coordinate space and magnitudes of electric field vectors. In conceptual blending theory, this conflation is described as a clash between the input spaces in the blend. We explore the benefits and drawbacks of this clash, as well as other features of this representation. This analysis is illustrated with examples from clinical problem-solving interviews with upper-division physics majors. We see that while these intermediate physics students make a variety of errors using this representation, they also use the geometric features of the representation to add electric field contributions and to organize the problem situation productively.
\end{abstract}

DOI: 10.1103/PhysRevSTPER.10.020112

PACS numbers: 01.40.Fk, 01.40.Ha

\section{INTRODUCTION}

One of the hallmarks of proficiency in physics is the ability to represent physical phenomena using various external representations such as diagrams, graphs, and equations [1]. Expert physicists flexibly use these various external representations both to communicate information about physical situations and as computational tools for solving problems. However, it is well documented that students often have difficulty creating and using these disciplinary representations [2-7].

We use conceptual blending theory [8] and distributed cognition [9] to understand the use of representations for problem solving. Specifically, we focus on how the material features of a representation might influence-both productively and unproductively-the reasoning of problem solvers. Conceptual blending is a general theory of cognition that aims to describe how people make meaning and has been used to describe some aspects of physics reasoning. Bing and Redish [10] have used conceptual blending theory to describe the relationship between mathematical knowledge and physics knowledge during problem solving. Wittmann

\footnotetext{
*egire@memphis.edu

Published by the American Physical Society under the terms of the Creative Commons Attribution 3.0 License. Further distribution of this work must maintain attribution to the author(s) and the published article's title, journal citation, and DOI.
}

[11] has used it to describe how students construct conceptual explanations for waves on a spring. Podolefsky and Finkelstein [12] use conceptual blending theory along with ideas from semiotics to explain how students make meaning of abstract representations of sound and electromagnetic waves. Distributed cognition seeks to locate cognitive activity in "a wider dynamical process of which the cognition of the individual is only a part" ([9], p. xiii). We extend the use of conceptual blending theory to study how students create and use external representations for performing computations in physics, and we draw on distributed cognition to understand how representations can support and restructure cognitive tasks. In this paper, we focus on students' use of the vector-arrows representation of electric field to add two fields together.

Some models of how students interpret external representations in physics have been proposed. Elby [13] suggests that sometimes a compelling visual attribute of a representation can activate an intuitive knowledge element he calls "what-you-see-is-what-you-get" (WYSIWYG). This knowledge element then causes students to make a more literal interpretation of the attribute. Alternately, Podolefsky and Finkelstein [12] have developed a model of analogical scaffolding to explain how students interpret new representations. The new representation acts as a sign that activates understandings of what is represented. Students draw analogies with other similar, familiar representations to inform their understanding of the new representation. This model suggests that instructors can 
carefully scaffold the introduction of representations so that elements of understanding that are productive for interpreting the new representation are recruited. Podolefsky and Finkelstein [14] demonstrated that carefully scaffolding concrete and abstract representations of waves on a string, sound waves, and electromagnetic waves leads to more productive student reasoning about these representations.

Both of these models focus on how students interpret external representations that are encountered, but not generated, by the student, and they both examine conceptual understandings that are activated by representations. Our analysis focuses on students' construction of an external representation for solving a physics problem. We look at the process of how students draw representations and how the material features of the representations support or hinder student reasoning. Additionally, although both the WYSIWYG and analogical scaffolding models include some discussion of the role of the features of the representations, these discussions are limited. The WYSIWYG model discusses only visually compelling features of the representation. Analogical scaffolding includes only representational features as signs that activate conceptual knowledge. Our analysis considers other roles of representational features, particularly with regard to how material features support the functional use of representations for computations.

The goals of the paper are to (1) demonstrate how distributed cognition and conceptual blending theory can be used to give an account of how people make meaning of external representations in physics through the specific example of the vector-arrows representation of electric field and (2) discuss some of the roles of the material anchors of this representation as illustrated by some examples from students performing a superposition of two electric fields task. This paper focuses on a particular representation (electric field vector arrows) used for a particular computation (a superposition task) in order to illustrate how the material features of a representation may interact with student problem solving. We also include some more general discussion of how material features of representations support computation in physics.

We begin by describing conceptual blending theory and the elements of the theory that are relevant for our analysis, particularly the consideration of the material features of representations. Next, we discuss how the vector-arrows representation of electric fields constitutes a conceptual blend and how the material features of the representation support a superposition task with illustrative examples from student interviews. Finally, we discuss the implications of this analysis for future research and instruction.

\section{CONCEPTUAL BLENDING THEORY WITH MATERIAL ANCHORS}

In thinking about representations and their use by physicists and students, we draw on the perspectives of conceptual blending theory [8] and distributed cognition [9]. Conceptual blending is a general theory of cognition aimed at describing how people make meaning out of situations, language, and other representations of information. Distributed cognition provides an account for the role of external entities (including tools, other people, and representations) in cognition.

In conceptual blending theory, meaning is made by selectively projecting elements of separate input mental spaces onto a blended mental space, or blend, from which new meaning emerges (Fig. 1). Each input mental space has an organizing frame that specifies the relationships among the elements within the space. Input mental spaces are connected to each other through vital conceptual relations, such as time, space, identity, analogy, etc., that lead to correspondences between the elements of each space. The elements and organizing frames of the input spaces can be selectively projected into the blend in a variety of combinations. In a double scope blend, both input spaces contribute elements of their organizing frames to the blended space. The dual organizing frames can give rise to a clash between vital conceptual relations ([8], p. 125), which must be negotiated during the interpretation and use of double scope blends. We will demonstrate that the vector-arrows representation of electric fields arises from a double scope blend with a clash on the vital conceptual relation of space. The clash may lead to student difficulties in using the vector-arrows representation for electric field.

When a conceptual blend involves a material object (or a collection of objects), it is a material anchor of the blend. For example, a line of people and the concept of sequenced individuals can be combined to form a blended space corresponding to a queue [15]. In this case, the line of people is the material anchor. The existence of this material anchor facilitates certain operations, like determining who will be the fourth person to receive service. The blend arises

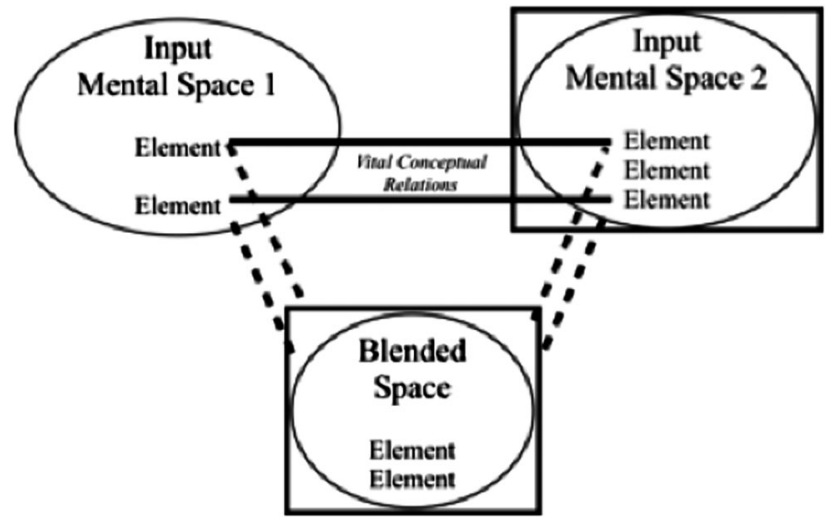

FIG. 1. Generic network map for a conceptual blend. Ovals indicate input and blended mental spaces. Solid lines indicate correspondences between input spaces. Dotted lines indicate elements that are projected into the blended space. Rectangles indicate a space that includes a material anchor. 
from the combination of the concept (sequenced individuals) with the material anchor. Without the material anchor of the line of people (i.e., if the people were haphazardly milling about), or without the conceptual structure (the sequence of individuals), these operations would be more difficult to accomplish. While Fauconnier and Turner [8] (like Podolefsky and Finkelstein) emphasize the "sign" aspects of material anchors as pointers to the concepts that they represent, such as bills or coins in the case of money, or word forms in the case of writing, Hutchins [16] emphasizes the stabilizing and computational functions of material anchors to support cognition. For representations in physics, the sign aspect of material anchors is certainly important, but our primary interest is in how the material anchors of a representation support cognition, particularly computation.

External representations can be thought of as artifacts having both material (physical) and ideal (conceptual) aspects [17]. In conceptual blending theory, these artifacts are material anchors. Distributed cognition emphasizes an artifact's ability to support cognition and restructure the tasks associated with cognitive activity. In the case of a graph, the material features include the ink marks that make up the graph and the surface on which it appears (e.g., paper or whiteboard). These features both enable and constrain possibilities, and can therefore help us understand how representations can support or constrain students' thinking. For instance, once drawn, a graph is stable, thus freeing working memory for other tasks. However, due to the fact that paper is two dimensional, objects and functions can only be shown in cross section or perspective, which requires cognitive processing to interpret. Representations can also transform computational tasks [9]. For example, consider the different cognitive demands of computing a definite integral using an algebraic representation and a graphical representation. As Hutchins puts it, "each tool presents the task to the user as a different sort of cognitive problem requiring a different set of cognitive abilities or a different organization of the same set of abilities" ([9], p. 154). In this way, material features affect the computational power and the pedagogical affordances of a representation.

To an experienced physicist, the following description of the vector-arrows representation of the electric field may seem pedantic or obvious. This is often the case with conceptual blends, which become so familiar and automatic that we take their complexity and sophistication for granted. As Fauconnier and Turner put it, "Because the brain does this instantly and unconsciously, we take the construction of meaning for granted" ([8], p. 5)

\section{ELECTRIC FIELD VECTOR MAP CONCEPTUAL BLEND}

We propose that the vector-arrows representation of an electric field in a region of space arises from a conceptual blend we call the electric field vector map (Fig. 2). The input mental spaces for this blend are the individual electric field arrows space and the spatial map space. Each of these mental spaces are themselves blends of other mental spaces.

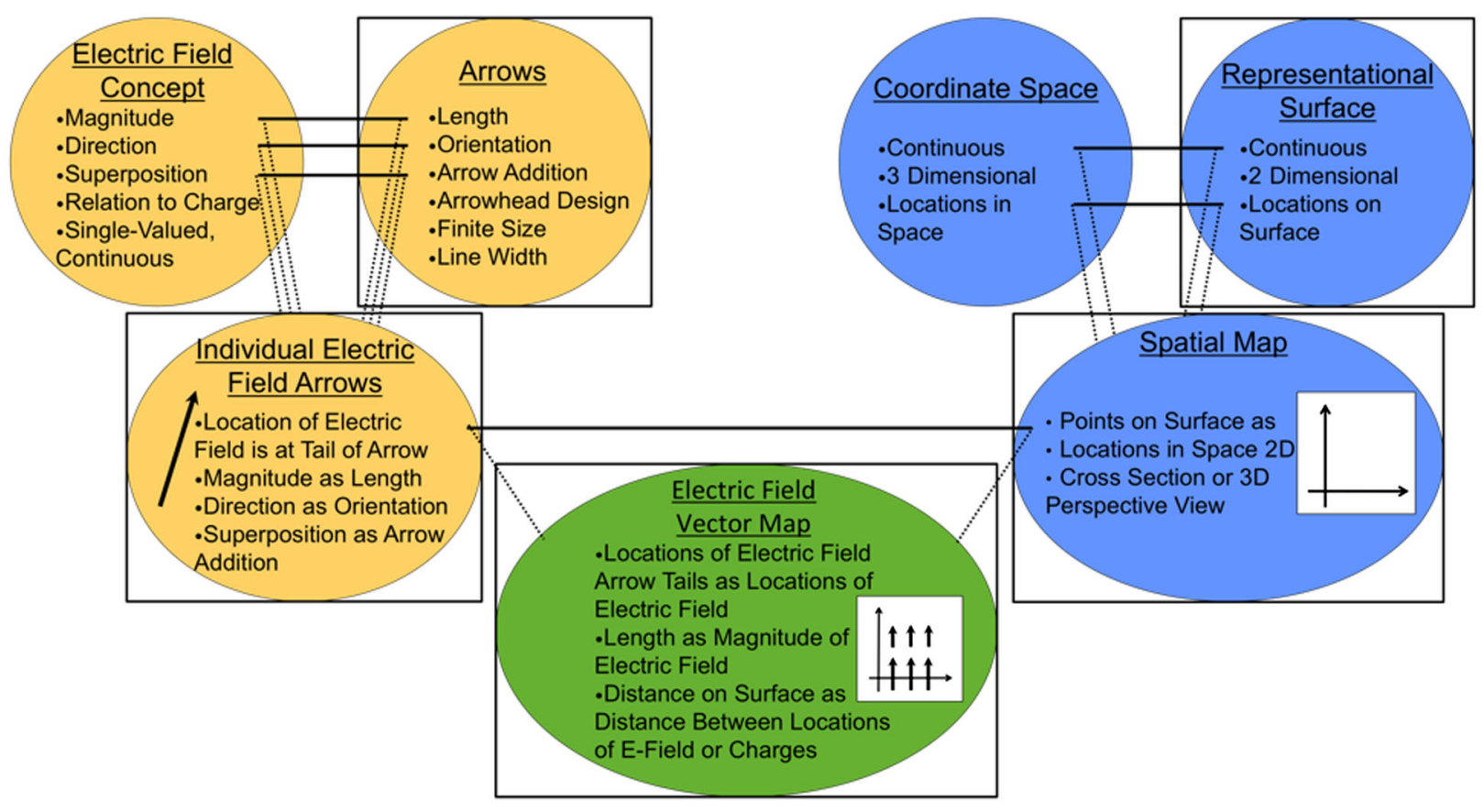

FIG. 2. Network for the double scope electric field vector map blend. The electric field vector map is a blend of the individual electric field arrows and spatial map input spaces, which are each blends of other input spaces. 
The individual electric field arrows space is a blend of an electric field concept space with an arrows space. The electric field concept space includes elements associated with the physics and mathematics of the electric field such as magnitude, direction, combination through superposition, relation to source charges, and units. Elements in the arrows space include length, orientation, and the design of the arrow. A drawn arrow is a material anchor for the arrows input space. Many elements in the arrows space have corresponding physical attributes (location, length, and orientation); the physical arrows also have persistence (until they are erased). Elements from the electric field concept and arrows spaces are projected into the blended individual electric field arrows space. In this blended space, the strength of the field corresponds to the length of the arrow, the direction of the electric field corresponds to the orientation of the arrow, and superposition corresponds to geometric addition of vector arrows. The material anchor of this space is the arrow. The organizing frame of this individual electric field arrows space is a vectors frame, where the important features are specifically related to properties of vectors, namely, the magnitude (amount), the direction, and operations like addition.

Likewise, the spatial map space is a blend of the coordinate space and representational surface spaces. Coordinate space is continuous, three dimensional, and includes locations. Representational surface is an input space with a material anchor such as a piece of paper or whiteboard, is continuous, two dimensional, and includes locations on the surface. In the spatial map blend, physical space is mapped onto a representational surface and a subset of points in physical space corresponds to locations on the representational surface. A coordinate system could be imposed to aid the correspondence, and sometimes the objects in real space, like a charge distribution, are also represented. In this blend, the material anchors are the representational surface and the drawings of the coordinate axes and other objects. The organizing frame is a mapping frame.

In the electric field vector map blend, these two input spaces (individual electric field arrows and spatial map) are combined to represent the electric field in a region of physical space: physical space is mapped onto the representational surface and the electric field in the physical space is represented as arrows on the surface. The material anchor for this blend provides stability and captures the geometric connection of the electric field to physical space and charged objects. In subsequent sections, we show that during problem solving, these features support connection to other representations and the identification of regions of interest.

The electric field vector map blend is a double-scope blend. Both the mapping frame from the spatial map space and the vectors frame from the individual electric field arrows space are necessary for correctly interpreting the blend.
For the discussion that follows, it is useful to note how algebraic representations are connected to the material features of their representations and to contrast this with the preceding discussion of the electric field vector map. Materially, an algebraic representation consists of symbols drawn on a surface. What is the significance of this physical form? It provides stability, facilitating elaborate manipulations. Elements of the representation are distinct from each other ("individuation," according to Hutchins). However, the material aspects of the representation are relatively unimportant. The spacial mapping onto a physical surface, so central to the electric field vector map blend, has a more limited significance in an algebraic representation (examples might include grouping similar terms or using a conventional ordering of elements).

\section{SOME COMPUTATIONAL IMPLICATIONS OF AN ELECTRIC FIELD VECTOR MAP}

At this point, we would like to discuss some of the computational implications of the material features of the electric field vector map blend. To this end, we present episodes from a set of problem-solving interviews that illustrate the computational affordances of the material features of the electric field vector map blend. After developing the theoretical analysis presented above, we designed an interview task where the use of and coordination between representations would greatly facilitate the solution. We will begin by describing these interviews and the interview task, so that these examples make sense to the reader.

\section{A. Problem-solving interviews}

We interviewed $n=8$ physics majors at Oregon State University (OSU) who participated in the Paradigms in Physics program $[18,19]$. The interviews were semistructured in nature and students were asked to think aloud as they were performing the interview tasks [20]. The goal of these interviews was to explore how students used various representations to perform a computation (in this case, a superposition task). OSU students were chosen because the Paradigms in Physics program emphasizes the use of and coordination between representations, as well as students' explanation of their thinking (through small group and whole class discussions). Thus, we expected these students to be familiar with the type of tasks included in the interviews and comfortable articulating their thinking. We also expected that advanced undergraduate students would be familiar with the conventions of various representations of electric field and that their use of the representation might highlight the role of the material features. The interviews were videotaped and segments of interest were transcribed for analysis.

As a warm-up exercise to remind students about various representations of electric fields, students were first asked 
to represent the electric field due to an infinite charged sheet in as many different ways as they could. After the students exhausted their own suggestions for representations, the interviewer suggested additional representations, including vector arrows, field lines, equations, and graphs of the electric field component versus spatial coordinate to ensure that all of the representations of interest to us were discussed. The analysis presented in this paper focuses on the students' use of electric field vector arrows, although we will also briefly discuss the use of the vector arrows to aid in generating equations for the electric field.

An important feature of the physics for the primary interview task is that the electric field due to a charged infinite sheet is uniform and perpendicular to the sheet (away from the sheet in the case of a positive charge density). Our primary goal for the interview was in exploring how students use the representations for computation, not to determine if they were familiar with this particular feature of the physics. Therefore, in the warm-up task, if the student did not mention this feature of the physics, the interviewer did. We hoped that this would increase the likelihood that students would incorporate the correct physics into their solutions for the primary task, and that student errors would be primarily associated with the use of the representations.

For the primary interview task, the students were verbally asked to consider two charged infinite sheets intersecting at a $45^{\circ}$ angle, with one sheet in the $z=0$ plane. The students were then asked how they would represent the electric field due to the sheets. The computation in this task is adding the electric fields from each sheet. All of the students assumed that the sheets were positively charged with uniform and equal charge densities. The examples presented in this paper focus on the students' use of the electric field vector-arrows representation during this primary interview task.

Video recordings of the interviews were viewed and discussed by both of the authors. We identified episodes where the material features of the representations appeared to influence the students' work. These episodes were then transcribed for further discussion until both authors reached an agreement on how to interpret the role of the material features. The student work presented in this paper were selected as particularly illustrative examples (i.e., extreme cases [21]) of how the material features of the vector-arrows representation may have influenced the students' creation and use of the representation for adding two electric fields.

\section{B. Solution to the primary interview task}

Assuming that the charge on each sheet is positive and uniformly distributed, the electric field due to each sheet is constant in magnitude, perpendicular to the sheet, and points away from the sheet on each side of the sheet. The two sheets establish four spatial regions, and the net electric field in each region is uniform (Fig. 3)

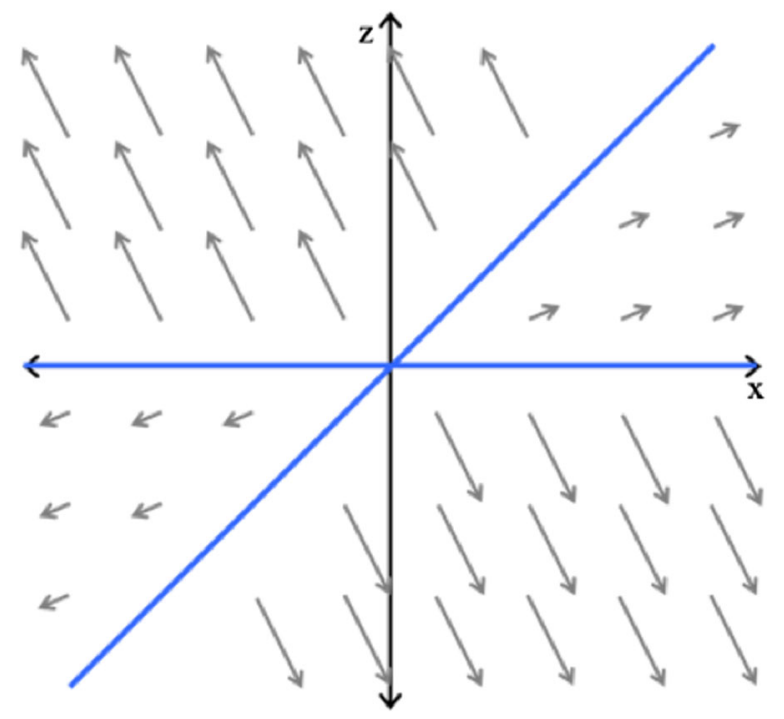

FIG. 3. Possible solution for the primary interview task. The cross sections of the charged sheets are represented by thick blue lines. The electric field is represented by gray arrows. The electric field is uniform in each of the four regions established by the sheets.

\section{SOME WAYS THAT THE ELECTRIC FIELD VECTOR MAP SUPPORTS PHYSICAL REASONING}

\section{A. Dual role of space may cause confusion}

For example, one student, Jim, started his vector-arrows representation by drawing two electric field arrows, one near and perpendicular to each drawn charged sheet. He indicated that each of these arrows represented the electric field due to the nearest sheet. He then drew two new arrows, smaller than the first two, near the middle of the region. These new arrows were drawn head to tail and added together. He then drew more arrows along the line that bisects the region and some arrows near the intersection of the sheets.

At the locations near the intersection, the arrows are drawn as being short, but at locations farther away from the intersection, the arrows are drawn longer (Fig. 4). This pattern is puzzling because it is inconsistent with the field from each sheet being uniform (which Jim correctly articulated in the first part of the interview).

One possible explanation is that Jim has drawn the arrows so that they do not intersect the lines representing the charged sheet. This suggests that the spatial map is interfering with the length's interpretation as magnitude in the electric field vector map; this can be understood in terms of blending theory [22] .

The material features of the electric field vector map representation introduce an ambiguity in the meanings of space on the representation: the two-dimensional surface and the use of an arrow representation introduce ambiguity in the interpretation of spatial extent. In the language of 


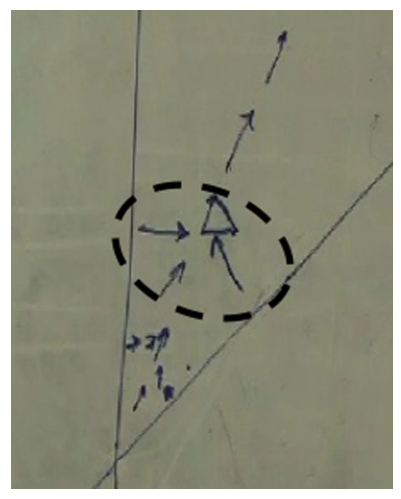

FIG. 4. Jim's electric field vector map for two charged infinite sheets. The long lines represent the charged sheets. Jim first drew the arrows (circled) representing the electric fields from each sheet and then he added them head to tail as shown. Then he drew the other arrows (outside our circle) representing the combined electric field due to the two sheets. The smallest arrows lie closest to the intersection, and the arrows nearest the sheets are perpendicular to the sheets.

conceptual blending theory, this blend has a clash ([8], p. 125) on the vital conceptual relation of space. In the spatial map space, the points correspond to locations in real space, and distances between points correspond to physical distances. In the individual electric field arrows space, the distance between the tip of the arrowhead and the end of the arrow tail corresponds to the magnitude of the electric field. In the blend, distance is ambiguous; distance can represent either physical lengths or electric field strengths. Furthermore, while a vector arrow represents the electric field at a single point, the arrow has a finite extent. If interpreted in the spatial map space, the single electric field value is associated with a continuum of points along the span of the arrow.

Considering this dual role of space in these electric field vector map representations of electric field provides a plausible explanation for Jim's drawing (Fig. 4). His drawing is consistent with the (unconventional) idea that the electric field represented by the arrow exists along the entire length of the arrow, not just at the end of the tail [23]. As a result, the sheets artificially confine the lengths of the electric field arrows. In drawing the arrows short, the arrows do not "carry" electric field across a sheet and into regions where the electric field is known to be different. As a result, the length of the arrow, which has no spatial meaning (its meaning is field magnitude) is constrained by physical space in the electric field vector map, so physical crowding has an impact on the magnitude. Unfortunately, Jim does not provide any direct evidence of this reasoning during the interview.

Resolving this ambiguity or conflict of the meaning of spatial extent on this representation is a matter of disciplinary convention. The normative resolution is for the arrow to represent the electric field at the location of the end of the tail of the arrow and for the relative lengths of the arrows to indicate the relative strengths of the electric fields. This resolution allows for overlapping or intersecting electric field arrows, or electric field arrows that intersect with other objects (like the charged sheets). The material anchor (the marks on a page that we interpret as an arrow) supports this approach, since intersecting and overlapping marks can easily be drawn. In contrast, if one wanted to prevent the arrows from crossing, the representation's material features offer no help. This is exactly the case with the field line representation, where the marks representing field lines should not cross (because it would indicate a multivalued field at that location), but the material features of the field line representation do nothing to prevent it. Crossing field lines are in fact a common error by students [24].

Another consequence of this dual role of space in the electric field vector map blend is the need for sampling. One cannot draw an arrow at every point in space even though the electric field exists at every point - the arrows would overlap so as to be unintelligible and it would take an inconveniently long time to draw. Therefore, only a subset of points is chosen, and arrow lengths are drawn with an arbitrary global scaling factor that is typically chosen so that the arrows do not overlap.

Some physicists use a different convention for indicating the magnitude of the electric field in the electric field vector map representation in that they hold the length of all arrows constant and allow the arrow's transparency to represent the magnitude of the electric field (with more transparent arrows indicating a weaker electric field). This convention is usually employed with computer-generated representations [25]. Although this convention makes choosing a spatial sampling rate easier and reduces the chance of overlapping electric field arrows, it no longer supports easy computation of vector addition. However, this tradeoff makes sense when computation is handled by the computer application.

\section{B. Material features of the electric field vector map support superposition computations}

A further consequence of this stability for comparisons is the ability to add electric field vectors geometrically (i.e., "tip to tail"). This is possible because the "linearity" of the representational space matches the linearity of vector addition. In this way, the representation encodes the computational process of superposition. In contrast, in an algebraic representation, one adds "component wise," which requires decomposing each vector into components (using a consistent coordinate system), adding the corresponding components, then reconstructing a vector from the result. With vector arrows, much of the computation is built into the representation; one does not need to consider components or even designate a coordinate system ([9], pp. 147-154). However, with vector arrows one obtains a 


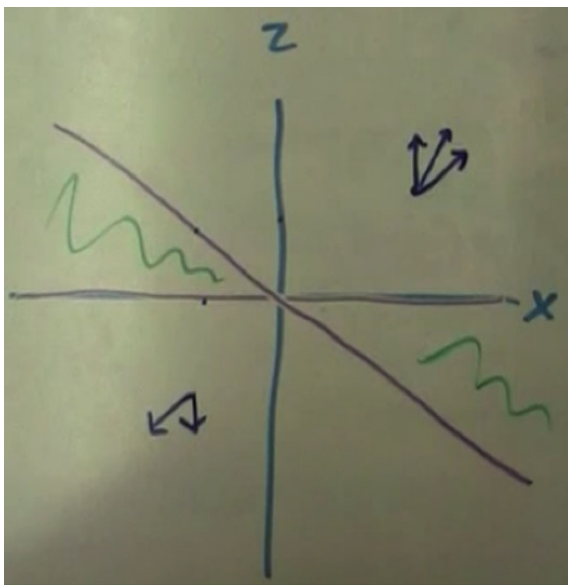

FIG. 5. Anne's electric field vector map drawn while considering the electric field in the two nonshaded regions defined by the charged sheets which are drawn in purple ink. At the top right, Anne has drawn arrows representing the electric field at a point due to each of the charged sheets and the net electric field. At the bottom left, she has only drawn arrows corresponding to the electric field at a point from each of the charged sheets.

geometric solution, while algebraic representations provide a quantitative form. More powerfully, the coordinated use of and translation between the two representations can facilitate computation in both.

To illustrate this, let us consider Anne's solution to the interview task. She first draws two arrows, each representing the electric field from one of the charged sheets, at a point in the upper left region defined by the charged sheets (Fig. 5). She then draws a longer arrow that bisects the first two, representing the net electric field at that point. It is interesting to note that this longer net electric field arrow is in fact too short to be the vector sum of the other constituent electric field arrows and can be considered an error in Anne's solution. This inattention to detail may indicate that Anne does not take this vector arrow approach seriously as a solution method, but instead is using the method to qualitatively sketch the solution.

Next, she tries to use trigonometry to determine the magnitude of the field (Fig. 6).

Um, so it'll be pointing up at ... 70 degrees from the $x$-axis with a magnitude of ... let's see ... so if we have um $E_{0}, E_{0} \ldots$ this would be the same as $E_{0} E_{0} u m$... let's say we know that this is 22.5 ... relations here, um, sine is opposite over adjacent, sine of 22.5 equals opposite, no no no, opposite over adjacent, that's tangent. Um ... that's, that only works for right triangles, that's all right. Um, this one's going to be 180-45 is 135. Um, there's a relationship here that I can't remember. Um, you'd be able to get the magnitude, we'll call it $E_{n}$ for now, um, using that I know, I know with three angles and two of the sides you could find the third side. I just can't think of which relationship it is right now.

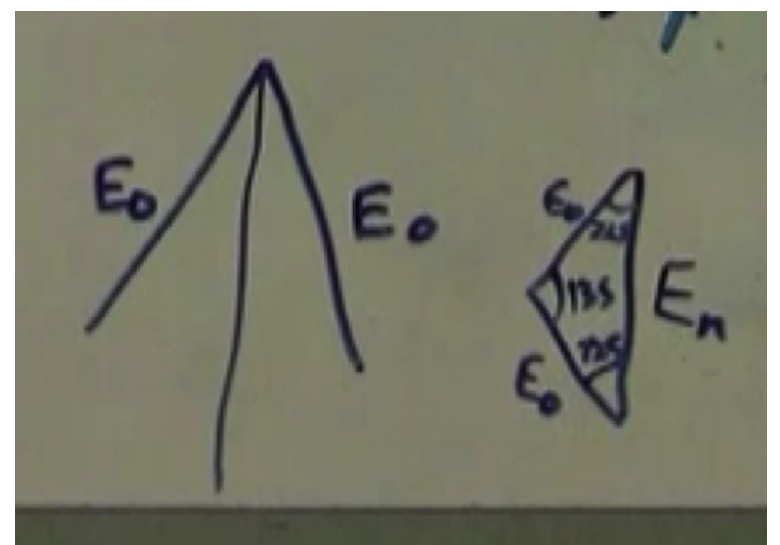

FIG. 6. Detail of Anne's calculation of the net electric field for the lower left region using the vector-arrows representation (without having drawn the arrow heads). The lines labeled " $E_{0}$ " represent the electric field vectors from each charged plane. The net electric field is represented by the unlabeled line on the leftmost diagram and the line labeled " $E_{n}$ " in the rightmost diagram.

Anne has quickly identified the vector sum of the two component electric fields without reference to a coordinate system, and then spends some time trying to figure out the magnitude of the net electric field using trigonometry. She uses alphanumeric symbols to label the lengths of the lines and the angles, but does not manipulate these symbols algebraically. This work is physically embedded in and conceptually supported by the vector-arrows representation. Although she is ultimately unsuccessful, she expresses certainty that she could find the magnitude of the net electric field if she remembered the appropriate trigonometric relationship.

She then immediately, without prompting from the interviewer, switches to an algebraic representation of vector components (Fig. 7):

Or I suppose, I guess you could write it as, you know, $E_{0}$ ah ... that was i $j k$ that I was using, so it's going to be in the, pointing in the, that direction, which is going to be $k$ hat or whatever I was calling it, plus $E_{0}$ in the, so, if we divide this one up into components [draws an arrow in the lower right corner], 45-45-90, you end up with um $E_{0}$ over root 2 in the $i$ hat, ah $i j k$ yeah, $i$ hat plus $E_{0}$ over root $2 k$ hat. Um, and then you combine those terms.

The addition of the two constituent electric field arrows (drawing the appropriate arrows) is fast and seemingly effortless for Anne. She quickly identifies the magnitude of the angle of the net electric field with respect to her coordinate system but has some difficulty in describing the magnitude of the electric field relative to the magnitude of the constituent fields. In contrast, she can determine the relative magnitude of the electric field components using an 


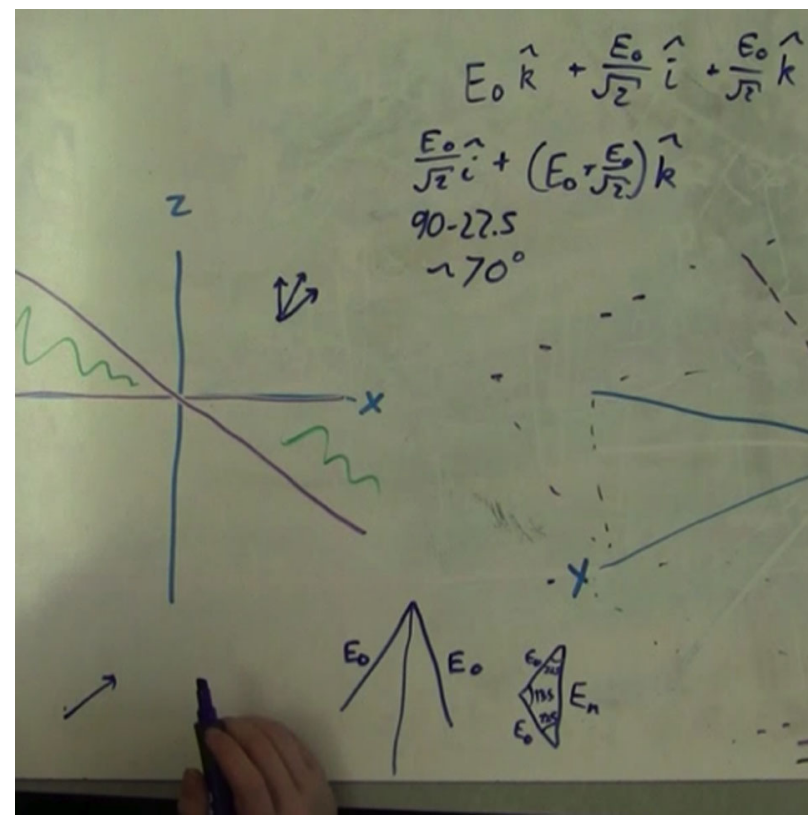

FIG. 7. Overview of Anne's board while performing the superposition task, using both the vector-arrows representation (bottom, center) and an algebraic representation (upper right).

algebraic representation. However, in writing down an algebraic representation of the net electric field, Anne refers back to her drawing several times to compare the vector arrows that she has drawn with her coordinate system.

These examples illustrate how the representation, as a conceptual blend with material anchors, changes what is required to do the computation in the primary interview task. Some aspects are facilitated by the vector arrows representation while others are facilitated by an algebraic representation. The two representations support each other, and coordination between the two aids the computational task.

The electric field line representation provides a contrasting example of how material features can support computation. In the field line representation, where the relative magnitude of the field is represented by the relative spacing of lines, superposition computations are much more difficult. However, other computations are easier, for instance, determining whether the electric flux through a surface is zero. In the case of electric flux, the material features of the representation (namely, the compensatory scaling of line density and a circle's circumference) transform the computation from a complex vector integration over the surface to simply comparing the number of field lines that enter and exit the surface.

The match between computational operation and material features is not inevitable; indeed, it helps explain why, of all the possible representations that could be used, physicists regularly employ the ones we do. For instance, consider a spherical whiteboard. In this case, it is sensible to map a spherical surface in physical space to locations on the representational surface, for instance, the surface of Earth represented on a globe. In the electric field vector arrows blend, the arrows would also be drawn in the curved space. The magnitude of the field can still correspond to the length of the arrow, but because the arrow is confined to the curved surface, the direction of the field becomes distorted as the arrow's orientation changes (relative to the tip) over the length of the arrow. The tip to tail process can still be performed, and is appropriate for addition of displacement vectors (i.e., airplane flights). But geometric addition of the vectors is not appropriate for electric field vectors in this representation.

\section{Space provides an organizational structure and stability that supports extrapolation}

As a map, an electric field vector map emphasizes spatial relationships and allows the student to visualize spatial patterns or important symmetries. When the charge distribution is included on the map (which is very often the case), the electric field vector map highlights the spatial relationships between the sources of electric field and the locations where the field is represented or evaluated.

For example, when trying to find the net electric field due to the two charged sheets, Anne draws the $x$ and $z$ coordinate axes in blue ink and the charged sheets in purple ink (Fig. 5). She then shades in green ink two regions defined by the charged sheets, saying,

So at any given point outside the two ... I'll get to the green section in a bit because it's going to be significantly harder. Um, for any given point outside of these two [green regions], the electric field is going to be a combination of pointing away from, so let's go so let's say at this point [draws a point in the upper right region], there's going to be field pointing from this infinite plane this direction [draws arrow upward] and field, er, a field line from this one that goes at 45 [draws an arrow at a 45 degree angle] um which means that overall any point outside of the wedges is going to be, 45 , 90, is going to be somewhere in there, half of whatever 45 is-22.5 [draws a third arrow bisecting the first two]. So, it's going to be pointing up at approximately that angle which is like $90-22.5$ which is 70 give or take.

At this point, Anne has used her electric field vector map to identify and distinguish different regions of interest and determined the approximate orientation of the electric field in one of these regions. She then goes on to identify the net electric field in each of the four regions of interest. For the first two regions (upper right and lower left, Fig. 5), she determines the net electric field by selecting a point in the region, drawing the electric field 


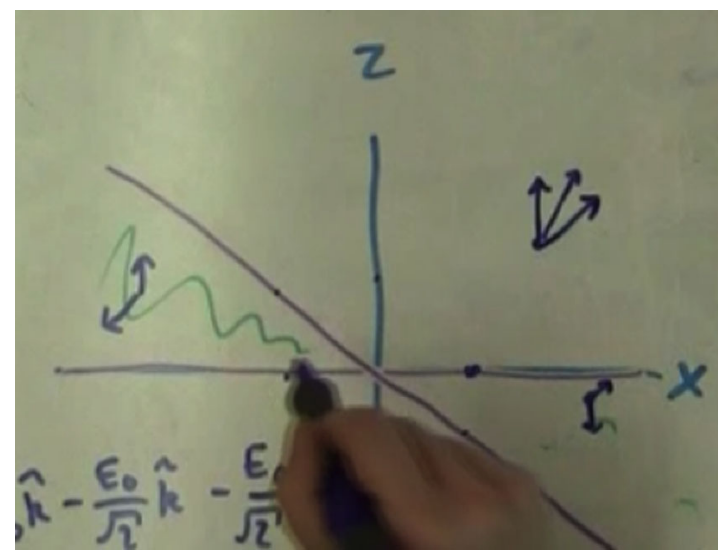

FIG. 8. Anne's electric field vector map after determining the electric field in all four regions of interest.

due to each charged sheet at that point, performing a geometric addition of the vector arrows, and then performing an algebraic addition of the electric field vectors. For the final two regions shaded in green, she does not perform the geometric addition, only the algebraic addition of the constituent fields (Fig. 8).

Using the electric field vector map, Anne quickly (and it seems effortlessly) identifies the four regions of interest to her and further identifies two of those regions as being "significantly harder" for her to think about than the others. In contrast, when she then tries to algebraically define the regions of interest, after she has identified these regions using the electric field vector map and without prompting from the interviewer, it is much more difficult for her to do, as indicated by several periods of quiet thinking and "guessing and checking" some algebraic statements. During this process, she clearly refers to her drawings of the charged sheets and coordinate axes by pointing to various features of these drawings. This task of identifying regions of interest is facilitated by the blend's mapping of physical space onto the representational space. In contrast, the algebraic representation alone lacks such a feature and does not as easily support the task.

As another example of the electric field vector map providing an organizational structure, six out of the eight [26] students began their electric field vector map by drawing vector arrows representing the electric field due to each sheet at points very near the source sheet (Fig 9, left). John draws a electric field vector map and explains:

So at this point I would just think that the, as far as representing the e-field, just the superposition principle, in the sense that I know that all the, um, that all of the, um, e-vectors coming from this field are the same magnitude pointing away. And all of the e-vectors from this, that are generated by this plane are the same but pointing in different directions. So, um, this would have

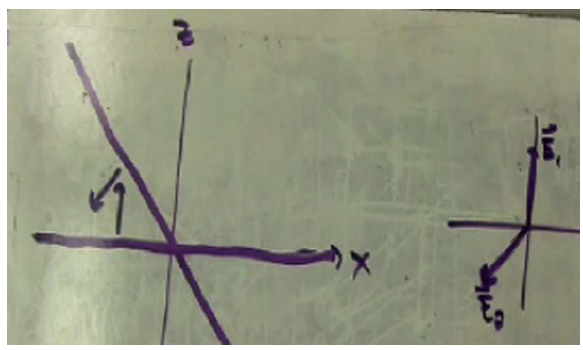

FIG. 9. An example of a student first drawing electric field arrows drawn near or on the lines representing the charged sheets (left), and then (when prompted to perform the addition) placing the electric field arrows tail to tail to find the combined electric field at an arbitrary point (right).

something like that and this would have something like that [draws two arrows, each perpendicular to one of the lines representing the charged sheets] and we would have to add the vectors together as far as pictorially would.

From John's statements, we know that the arrows that he has drawn do not represent the net electric field at different points; they each represent the electric field due to the nearest charged sheet only. John indicates that in order to find the net electric field, one would have to add the electric field vectors.

Although none of the students who drew the constituent electric field arrows in this way (rather than at the same point on the representational surface) articulated specific reasoning for doing so, we suspect that the proximity of these arrows to the drawn charged sheets helps the students associate the arrows with the field from the nearest sheet. From the material anchors perspective, we view the students as "borrowing" the proximity in representational space to signify a conceptual (causal) connection. The initial arrows may provide stability to support the drawing of other arrows farther away from the sources, where the association with the sources is not supported by spatial proximity and may therefore be more tenuous for the student.

Alternately, these students may be in the habit of first drawing electric field vectors at points near charged surfaces because the orientation of the electric field at these points is generally known to be perpendicular to the surface. For both of these explanations, the students are using the spatial features of the map to organize their ideas and to prepare for extrapolating the electric field to points farther away from the charged sheets.

The electric field vector map representation allows the student to visually compare the strengths and orientations of the field at different locations. The drawing provides a stable model of the electric field that stores and displays important spatial relationships. In other words, the external representation takes on some of the cognitive effort of "remembering" to facilitate reasoning. 


\section{DISCUSSION}

We see with the above example that the material features of the vector-arrows representation of electric field supports a superposition computation. The representation's stability, due to the materials used to construct it, allows students to organize, store, and display information about the electric field. This stability helps students to orient electric field vectors to charged objects while constructing the representation, extrapolate the electric field to new regions, and compare different electric field vectors for identifying patterns. This stability along with the representation's emphasis on spatial relationships facilitates comparisons between electric field vector arrows and coordinate axes, which in turn facilitates the translation from a vector-arrows representation to an algebraic representation of the electric field.

Furthermore, we have discussed how the material features of other representations (algebraic, field lines) differently support the same superposition computation. An algebraic representation supports a more precise, quantitative addition and description of the net electric field. Field lines do not easily support superposition because the computation is not "built in" to the material anchor for the representation.

We have used conceptual blending and distributed cognition theory as an explanatory framework for understanding students' use of external representations through the example of the vector-arrows representation of electric fields. Based on our analysis, we believe that student errors in using representations are most likely to arise from the following.

(1) Clashes on vital conceptual relations between input mental spaces-when a particular feature has two possible meanings on the representation, such as when length could mean either the distance between points or the magnitude of the electric field. Another example is when plotting the energy eigenstates of a quantum system on an energy diagram, the vertical axis often takes the dual role of representing the amount of energy associated with the eigenstate and the amplitude of the real part of the wave function,

(2) Clashes on vital conceptual relations between input mental spaces-when a material anchor is used to represent a quantity that is of a different type, such as when velocity is represented with a spatial coordinate on a kinematics graph $[13,27,28]$,

(3) Inattention to or unfamiliarity with the disciplinary conventions of how information is encoded in the material features of the representations, such as the relative length of the arrows representing the relative magnitude of the electric field, as in the case of Anne in Fig. 5, or including third law pairs of forces on a free-body diagram.

Empirical studies are needed to verify these predictions and explore the sources of student errors in using external representations.

In this view, the instructional strategy associated with analogical scaffolding [12] is effective because it is a structured process for helping students resolve a clash on vital conceptual relations. For populations that are likely to be familiar with the basic disciplinary conventions of the representation, we would expect that errors in using the representations would primarily be due to a clash on vital conceptual relations. In such cases, appropriate analogical scaffolding may be helpful.

Recently, the theoretical construct of "nesting" has been developed to explain how people represent many different ideas using limited physical space $[29,30]$. When a graphic is "nested," space in different regions has different meanings. Close et al. [29] describe the electric field vector map as a nested representation because the location of the end of an arrow corresponds to a physical location in space, but the electric field arrow should be interpreted with respect to an origin in a local electric field space. Close et al. argue that nested diagrams can become problematic if the learner is not cued to expect a "transition boundary of meaning" or if the "partition of meaning" is violated (e.g., when electric field arrows intersect). From the perspective of conceptual blending, we see nesting as a description of the particular clash on vital conceptual relations that occurs when a representation uses multiple meanings for spatial extent. Cuing learners helps them to anticipate and resolve these clashes. Instructors can aid learners by identifying nested representations that only weakly cue transition boundaries of meaning and then scaffolding the learner's interpretation of these nested graphics.

When empirical studies that identify student difficulties for specific representations are lacking, instructors might anticipate likely areas of student difficulties by looking for a clash on vital conceptual relations. Instructors need not go through a detailed analysis (like the one presented in this paper), but might look for the following:

- representational features that have two potential meanings (such as length meaning both distance between points and magnitude of electric field) and

- representational features whose material features do not match what is being represented (such as a magnitude of an electric field being represented by a distance between field lines, with less space corresponding to a greater magnitude).

\section{CONCLUSION}

We have described the vector-arrows representation of electric field as arising from a conceptual blend and have illustrated how the material features of this representation support student reasoning about the superposition of electric fields. Mapping physical space onto a surface supports making comparisons between electric field vectors at various locations, orienting electric field vectors with respect to charged objects, and comparisons between multiple electric field vectors, which, in particular, aids vector addition. Spatial extent on the representation is ambiguous, denoting both extent in physical space and the magnitude of the electric field. 


\section{ACKNOWLEDGMENTS}

We would like to thank the students who volunteered to be interviewed as part of this study, Corinne Manogue, Tevian Dray, and Mary Bridget Kustusch for their helpful discussions, and Brian Frank and Renee Michelle Goertzen for their feedback in preparing this manuscript. This work was supported by NSF Grant No. DUE 1023120.
[1] A. Van Heuvelen, Learning to think like a physicist: A review of research-based instructional strategies, Am. J. Phys. 59, 891 (1991).

[2] P. B. Kohl and N. D. Finkelstein, Patterns of multiple representation use by experts and novices during physics problem solving, Phys. Rev. ST Phys. Educ. Res. 4, 010111 (2008).

[3] D. Rosengrant, A. Van Heuvelen, and E. Etkina, Do students use and understand free-body diagrams?, Phys. Rev. ST Phys. Educ. Res. 5, 010108 (2009).

[4] T. Fredlund, J. Airey, and C. Linder, Exploring the role of physics representations: An illustrative example from students sharing knowledge about refraction, Eur. J. Phys. 33, 657 (2012).

[5] A. F. Heckler, Some consequences of prompting novice physics students to construct force diagrams, Int. J. Sci. Educ. 32, 1829 (2010).

[6] D. E. Meltzer, Relation between students' problem-solving performance and representational format, Am. J. Phys. 73, 463 (2005).

[7] P. B. Kohl and N. D. Finkelstein, Effects of representation on students solving physics problems: A fine-grained characterization, Phys. Rev. ST Phys. Educ. Res. 2, 010106 (2006).

[8] G. Fauconnier and M. Turner, The Way We Think (Basic Books, New York, 2002).

[9] E. Hutchins, Cognition in the Wild (MIT Press, Cambridge, MA, 1995).

[10] T. J. Bing and E. F. Redish, The cognitive blending of mathematics and physics knowledge, in Proceedings of the Physics Education Research Conference, Syracuse, NY, 2006 (AIP Press, Melville, New York, 2007), Vol. 883, pp. 26-29.

[11] M. Wittmann, Using conceptual blending to describe emergent meaning in wave propagation, in Learning in the Disciplines: Proceedings of the 9th International Conference of the Learning Sciences (ICLS 2010), edited by K. Gomez, L. Lyons, and J. Radinsky (International Society of the Learning Sciences, Chicago, IL, 2010), Vol. 1.

[12] N.S. Podolefsky and N.D. Finkelstein, Analogical scaffolding and the learning of abstract ideas in physics: An example from electromagnetic waves, Phys. Rev. ST Phys. Educ. Res. 3, 010109 (2007).

[13] A. Elby, What students' learning of representations tells us of constructivism, J. Math. Behav. 19, 481 (2000).

[14] N. S. Podolefsky and N. D. Finkelstein, Analogical scaffolding and the learning of abstract ideas in physics: Empirical studies, Phys. Rev. ST Phys. Educ. Res. 3, 020104 (2007).

[15] R. Langacker, Foundations of Cognitive Grammar (Stanford University Press, Stanford, CA, 1987).
[16] E. Hutchins, Material anchors for conceptual blends, J. Pragmatics 37, 1555 (2005).

[17] M. Cole, Cultural Psychology: A Once and Future Discipline (Harvard University Press, Cambridge, MA, 1998).

[18] C. A. Manogue, P. J. Siemens, J. Tate, K. Browne, M. L. Niess, and A. J. Wolfer, Paradigms in physics: A new upper-division curriculum, Am. J. Phys. 69, 978 (2001).

[19] C. A. Manogue and K. S. Krane, Paradigms in physics: Restructuring the upper level, Phys. Today 56, No. 9, 53 (2003).

[20] K. Anders Ericsson and H. A. Simon, Verbal reports as data, Psychol. Rev. 87, 215 (1980).

[21] M. B. Miles and A. Michael Huberman, Qualitative Data Analysis: A Sourcebook of New Methods (Sage Publications, Thousand Oaks, CA, 1994), 2nd ed.

[22] Unfortunately, there is no evidence from the interview to indicate whether or not this was the reason for the lengths of the arrows.

[23] E. Gire and E. Price, Graphical representations of vector functions in upper-division E\&M, in Proceedings of the Physics Education Research Conference, Omaha, NE, 2011 (AIP Press, Melville, New York, 2012), Vol. 1413, pp. 27-30.

[24] S. Törnkvist, K. A. Pettersson, and G. Tranströmer, Confusion by representation: On students' comprehension of the electric field concept, Am. J. Phys. 61, 335 (1993).

[25] An example of this convention is used in the PhET electric field hockey game, http://phet.colorado.edu/en/simulation/ electric-hockey, accessed 20 June 2013.

[26] When drawing the electric field vectors due to an infinite charged sheet in the warm-up exercise, all of the students began drawing electric field vector arrows at points very near or on the line representing the sheet before proceeding to points farther away.

[27] G. Leinhardt, O. Zaslavsky, and M. Kay Stein, Functions, graphs, and graphing: Tasks learning and teaching, Rev. Educ. Res. 60, 1 (1990).

[28] L. C. McDermott, M. L. Rosenquist, and E. H. van Zee, Student difficulties in connecting graphs and physics: Examples from kinematics, Am. J. Phys. 55, 503 (1987).

[29] H. Close, E. Close, and D. Donnelly, Nesting in graphical representations in physics, in Proceedings of the Physics Education Research Conference, Philadelphia, PA, 2012 (AIPPress, Melville, New York, 2012), Vol.1513,pp. 110-113.

[30] H. Close, C. Schiber, E. Close, and D. Donnelly, Students' dynamic geometric reasoning about quantum spin-1/2 states, in Proceedings of the Physics Education Research Conference, Portland, OR, 2013 (AIP Press, Melville, New York, 2012), pp. 93-96. 